\title{
ON A THEOREM OF HAZRAT AND HOOBLER
}

\author{
BENJAMIN ANTIEAU
}

(Communicated by Lev Borisov)

\begin{abstract}
We use cycle complexes with coefficients in an Azumaya algebra, as developed by Kahn and Levine, to compare the $G$-theory of an Azumaya algebra to the $G$-theory of the base scheme. We obtain a sharper version of a theorem of Hazrat and Hoobler in certain cases.
\end{abstract}

\section{INTRODUCTION}

Let $\mathbf{K}_{*}(X ; \mathcal{A})$ be the $K$-theory of left $\mathcal{A}$-modules which are locally free and finite rank coherent $\mathcal{O}_{X}$-modules and let $\mathbf{G}_{*}(X ; \mathcal{A})$ be the $K$-theory of left $\mathcal{A}$-modules which are coherent $\mathcal{O}_{X}$-modules.

We prove the following theorem.

Theorem 1.1. Let $X$ be a d-dimensional scheme of finite type over a field $k$, and let $\mathcal{A}$ be an Azumaya algebra on $X$ of constant degree $n$. Let $B_{\mathcal{A}}: \mathbf{G}_{i}(X) \rightarrow \mathbf{G}_{i}(X ; \mathcal{A})$ and $B_{\mathcal{A}}^{\mathbf{K}}: \mathbf{K}_{i}(X) \rightarrow \mathbf{K}_{i}(X ; \mathcal{A})$ be the homomorphisms induced by the functor $\mathcal{F} \mapsto$ $\mathcal{A} \otimes_{\mathcal{O}_{X}} \mathcal{F}$. Then,

(1) the kernel and cokernel of $B_{\mathcal{A}}: \mathbf{G}_{i}(X) \rightarrow \mathbf{G}_{i}(X ; \mathcal{A})$ are torsion groups of exponents dividing $n^{2 d+2}$;

(2) the kernel and cokernel of $B_{\mathcal{A}}^{\mathbf{K}}: \mathbf{K}_{i}(X) \rightarrow \mathbf{K}_{i}(X ; \mathcal{A})$ are torsion groups of exponents dividing $n^{2 d+2}$ if $X$ is regular.

Corollary 1.2. If $\mathcal{A}$ is an Azumaya algebra of constant degree $n$ over a scheme $X$ of finite type over a field $k$, then the base extension homomorphism

$$
B_{\mathcal{A}}: \mathbf{G}_{*}(X) \otimes_{\mathbb{Z}} \mathbb{Z}\left[\frac{1}{n}\right] \rightarrow \mathbf{G}_{*}(X ; \mathcal{A}) \otimes_{\mathbb{Z}} \mathbb{Z}\left[\frac{1}{n}\right]
$$

is an isomorphism

The theorem above should be compared to the following two theorems, which motivated us in the first place.

Theorem 1.3 (Hazrat-Millar [9]). If $\mathcal{A}$ is an Azumaya algebra of constant degree $n$ which is free over a noetherian affine scheme $X$, then

$$
B_{\mathcal{A}}^{\mathbf{K}}: \mathbf{K}_{i}(X) \rightarrow \mathbf{K}_{i}(X ; \mathcal{A})
$$

has torsion kernel and cokernel of exponents at most $n^{4}$.

Received by the editors April 4, 2011 and, in revised form, November 7, 2011.

2010 Mathematics Subject Classification. Primary 14F22; Secondary 19Dxx.

Key words and phrases. Azumaya algebras, twisted algebraic $K$-theory.

The author was supported in part by the NSF under Grant RTG DMS 0838697.

(C) 2013 American Mathematical Society 
Theorem 1.4 (Hazrat-Hoobler [8]). Let $X$ be a d-dimensional noetherian scheme, and let $\mathcal{A}$ be an Azumaya algebra on $X$ of constant degree $n$. Then:

(1) the kernel of $B_{\mathcal{A}}: \mathbf{G}_{i}(X) \rightarrow \mathbf{G}_{i}(X ; \mathcal{A})$ is torsion of exponent dividing $n^{2 d(d+1)+2}$, and the cokernel is torsion of exponent dividing $n^{4 d+2}$;

(2) the kernel of $B_{\mathcal{A}}^{\mathbf{K}}: \mathbf{K}_{i}(X) \rightarrow \mathbf{K}_{i}(X ; \mathcal{A})$ is torsion of exponent dividing $n^{2 d(d+1)+2}$ if $X$ is regular, and the cokernel is torsion of exponent dividing $n^{4 d+2}$ in this case;

(3) the kernel and cokernel of $B_{\mathcal{A}}^{\mathbf{K}}: \mathbf{K}_{i}(X) \rightarrow \mathbf{K}_{i}(X ; \mathcal{A})$ are torsion groups of exponent dividing $n^{2 d+2}$ if $X$ has an ample line bundle.

Since a degree $n$ Azumaya algebra is locally split by degree $n$ extensions, it is expected that the base extension map

$$
B_{\mathcal{A}}^{\mathbf{K}}: \mathbf{K}_{*}(X) \otimes_{\mathbb{Z}} \mathbb{Z}\left[\frac{1}{n}\right] \rightarrow \mathbf{K}_{*}(X ; \mathcal{A}) \otimes_{\mathbb{Z}} \mathbb{Z}\left[\frac{1}{n}\right]
$$

should be an isomorphism.

Here is a partial history of results and techniques in this direction.

Wedderburn's theorem [10] easily implies that $\mathbf{K}_{0}(k) \rightarrow \mathbf{K}_{0}(A)$ is injective with cokernel isomorphic to $\mathbb{Z} / m$, where $A \cong M_{m}(D)$ for a central $k$-division algebra $D$.

Green-Handelman-Roberts [5] proved that the map $B_{\mathcal{A}}^{\mathbf{K}}$ in equation (11) is an isomorphism when $\mathcal{A}$ is a central simple algebra of degree $n$ over a field. They used the Skolem-Noether theorem. That case has also been proven by Hazrat [7] using the fact that $A$ is étale locally a matrix algebra.

The theorem of Hazrat-Millar quoted above uses the opposite algebra. The theorem of Hazrat-Hoobler uses Bass-style stable range arguments and Zariski descent for $G$-theory.

Our result uses twisted versions of Bloch's cycle complexes. These twisted cycle complexes and the twisted motivic spectral sequence that relates them to $G$-theory are due to Kahn and Levine [11. It is possible that our result could be extended to essentially smooth schemes over Dedekind rings by a combination of the work of Kahn and Levine [11] and Geisser [4].

The following is an interesting corollary of our approach: there are natural filtrations of length $d$ on $\mathbf{G}_{i}(X)$ and $\mathbf{G}_{i}(X ; \mathcal{A})$ coming from 11]. The map $B_{\mathcal{A}}$ : $\mathbf{G}_{i}(X) \rightarrow \mathbf{G}_{i}(X ; \mathcal{A})$ respects the filtrations. We show that the induced maps on each of the $d+1$ slices have kernel and cokernel groups of exponent at most $n^{2}$.

It is worth mentioning two related functors on Azumaya algebras with values in abelian groups where the base extension maps are isomorphisms. Dwyer and Friedlander [3, 2.4, 3.1] showed that

$$
\mathbf{K}_{*}^{\text {ét }}(R ; \mathbb{Z} / m) \rightarrow \mathbf{K}_{*}^{\text {ét }}(R ; A ; \mathbb{Z} / m)
$$

is an isomorphism in some cases (all of which are Azumaya algebras over a noetherian ring), where $\mathbf{K}^{\text {ét }}$ denotes étale $K$-theory, as, for instance, in Thomason [12]. In this direction, it is possible to show (for instance, in the setting of Antieau [1]) that $\mathbf{K}^{\text {ét }}(X ; \mathcal{A})$ is an invertible object (in the sense of the Picard group) over $\mathbf{K}^{\text {ét }}(X)$ in the category of étale sheaves of $\mathbf{K}^{\text {ét }}$-module spectra on a scheme $X$.

Finally, Cortiñas and Weibel [2] proved that the base extension maps induce isomorphisms in Hochschild homology over a field $k$. 


\section{Twisted higher Chow groups And twisted $G$-theory}

Let $X$ in $\mathbf{S c h} / k$ be an integral $k$-scheme of finite type, and let $\mathcal{A}$ be a sheaf of Azumaya algebras on $X$ of rank $n^{2}$. The degree of $\mathcal{A}$ is defined to be the integer $n$. Let $\mathcal{E}$ be a left $\mathcal{A}$-module which is locally free and finite rank $n a$ as an $\mathcal{O}_{X}$-module. For generalities on Azumaya algebras, which as $\mathcal{O}_{X}$-modules are always locally free and of finite rank, see [6].

As in Kahn-Levine [11, define the cycle complex of $X$ with coefficients in $\mathcal{A}$ as follows. Let $S_{(s)}^{X}(t)$ denote the set of closed subsets $W \subset X \times_{k} \Delta^{t}$ such that

$$
\operatorname{dim}_{k} W \cap X \times_{k} F \leq s+\operatorname{dim}_{k} F
$$

for all faces $F$ of $\Delta^{n}$. Taking inverse images, $S_{(s)}^{X}(*)$ becomes a simplicial set. Let $X_{s}(t)$ denote the subset of irreducible $W$ in $S_{(s)}^{X}(t)$ such that $\operatorname{dim}_{k} W=s+t$. Define, for $t \geq 0$,

$$
z_{s}(X, t ; \mathcal{A})=\bigoplus_{W \in X_{s}(t)} \mathbf{K}_{0}(k(W) ; \mathcal{A}) .
$$

See [11, Definition 5.6.1]. Kahn and Levine show that this actually becomes a complex, $z_{s}(X, * ; \mathcal{A})$, and they define the higher Chow groups with coefficients in $\mathcal{A}$ as

$$
\mathbf{C H}_{s}(X, t ; \mathcal{A})=\mathrm{H}_{t}\left(z_{s}(X, * ; \mathcal{A})\right) .
$$

There are maps relating the complex $z_{r}(X, * ; \mathcal{A})$ to $z_{r}(X, *)$, the untwisted complex that computes Bloch's higher Chow groups. These are induced by the basechange map $B_{\mathcal{E}}$ and the forgetful map $F$ on $K$-theory:

$$
\begin{gathered}
B_{\mathcal{E}}^{\mathbf{K}}: \mathbf{K}_{0}(k(W)) \rightarrow \mathbf{K}_{0}(k(W) ; \mathcal{A}), \\
F: \mathbf{K}_{0}(k(W), \mathcal{A}) \rightarrow \mathbf{K}_{0}(k(W)) .
\end{gathered}
$$

The map $B_{\mathcal{E}}$ takes a $k(W)$-vector space and tensors with $\mathcal{E}_{k(W)}$ to produce a left $\mathcal{A}_{k(W)}$-module. The norm map $F$ simply forgets the $\mathcal{A} \otimes_{k(W)}$-module structure on a vector space. The kernels of both of these maps are zero.

Lemma 2.1. The compositions $F \circ B_{\mathcal{E}}^{z}$ and $B_{\mathcal{E}}^{z} \circ F$ are multiplication by na on $z_{s}(X, t)$ and $z_{s}(X, t ; \mathcal{A})$.

Proof. Indeed, since the rank of $\mathcal{E}$ is $n a$ as an $\mathcal{O}_{X}$-module, this follows immediately.

Corollary 2.2. The cokernel of $F: z_{s}(X, t ; \mathcal{A}) \rightarrow z_{s}(X, t)$ is a torsion group of exponent bounded above by $n^{2}$, and $B_{\mathcal{E}}^{z}: z_{s}(X, t) \rightarrow z_{s}(X, t ; \mathcal{A})$ is a torsion group of exponent bounded above by na.

Proof. In the first case, one always has $\operatorname{ind}\left(\mathcal{A}_{k(W)}\right) \mid n$, where $\operatorname{ind}\left(\mathcal{A}_{k(W)}\right.$ is the degree of the unique division algebra over $k(W)$ such that $\mathcal{A}_{k(W)} \cong M_{m}(D)$ for some $m$. Similarly,

$$
\left(\frac{n a}{i n d\left(\mathcal{A}_{k(W)}\right)^{2}}\right) \mid n a,
$$

so the second statement follows. 
Proposition 2.3. The kernels and cokernels of

$$
B_{\mathcal{E}}^{\mathbf{C H}}: \mathbf{C H}_{s}(X, t) \rightarrow \mathbf{C H}_{s}(X, t ; \mathcal{A})
$$

and of

$$
F: \mathbf{C H}_{s}(X, t ; \mathcal{A}) \rightarrow \mathbf{C H}_{s}(X, t)
$$

are torsion groups of exponent at most na.

Proof. This follows immediately from Lemma 2.1

Here is our main theorem. Theorem 1.1 follows from it by taking $\mathcal{E}=\mathcal{A}$.

Theorem 2.4. Let $X$ be a d-dimensional scheme of finite type over a field, and let $\mathcal{A}$ be an Azumaya algebra on $X$. Then, the kernels and cokernels of

$$
B_{\mathcal{E}}: \mathbf{G}_{r}(X) \rightarrow \mathbf{G}_{r}(X ; \mathcal{A})
$$

and of

$$
F: \mathbf{G}_{r}(X ; \mathcal{A}) \rightarrow \mathbf{G}_{r}(X)
$$

are groups of exponent bounded above by $(n a)^{d+1}$ for all $r \geq 0$.

Proof. Kahn and Levine [1] show that there is a convergent spectral sequence

$$
\mathrm{E}_{2}^{p, q}(\mathcal{A})=\mathbf{C H}_{q}(X,-p-q ; \mathcal{A}) \Rightarrow \mathbf{G}_{-p-q}(X ; \mathcal{A}) .
$$

There is also the motivic spectral sequence

$$
\mathrm{E}_{2}^{p, q}=\mathbf{C H}_{q}(X,-p-q) \Rightarrow \mathbf{G}_{-p-q}(X) .
$$

The functors $B_{\mathcal{E}}: \mathbf{G}(X) \rightarrow \mathbf{G}(X ; \mathcal{A})$ and $F: \mathbf{G}(X ; \mathcal{A}) \rightarrow \mathbf{G}(X)$ are compatible with these spectral sequences and the functors $B_{\mathcal{E}}^{\mathbf{C H}}$ and $F$ on higher Chow groups. Note that $\mathrm{E}_{2}^{p, q}=\mathrm{E}_{2}^{p, q}(\mathcal{A})=0$ whenever $q<0,-p<0$, or $q>d$.

We will prove the theorem for the kernel of the functor $B_{\mathcal{E}}$. The other cases are entirely similar. On the $\mathrm{E}_{\infty}$-page, the composition functor $F \circ B_{\mathcal{E}}^{\mathbf{C H}}$ is still multiplication by $n a$, so the kernels and cokernels of $B_{\mathcal{E}}^{\mathbf{C H}}$ on $\mathrm{E}_{\infty}$ are still of exponent at most $n a$. The spectral sequences abut to filtrations $F^{s} \mathbf{G}_{r}(X ; \mathcal{A})$ and $F^{s} \mathbf{G}_{r}(X)$, where

$$
\begin{gathered}
F^{(s / s+1)} \mathbf{G}_{r}(X ; \mathcal{A})=F^{s} \mathbf{G}_{r}(X ; \mathcal{A}) / F^{s+1} \mathbf{G}_{r}(X ; \mathcal{A}) \cong \mathrm{E}_{\infty}^{-r+s,-s}(\mathcal{A}), \\
F^{(s / s+1)} \mathbf{G}_{r}(X)=F^{s} \mathbf{G}_{r}(X) / F^{s+1} \mathbf{G}_{r}(X) \cong \mathrm{E}_{\infty}^{-r+s,-s}
\end{gathered}
$$

The filtration looks like

$$
0=F^{0} \mathbf{G}_{r}(X) \subseteq F^{-1} \mathbf{G}_{r}(X) \subseteq \cdots \subseteq F^{-d} \mathbf{G}_{r}(X)=\mathbf{G}_{r}(X) .
$$

The filtration $F^{s} \mathbf{G}_{r}(X)$ is of length $d$ by the vanishing statements. Let $z \in \mathbf{G}_{r}(X)$ be in the kernel of $F$, and let $\bar{z}$ be the image of $z$ in $E_{\infty}^{-r-d, d}$. Then, by hypothesis, $\bar{z}$ is in the kernel of $F$, so that $n a \cdot \bar{z}=0$. Thus, $n a \cdot z$ is contained in $F^{-d+1} \mathbf{G}_{r}(X)$. Continuing in this way, we see that $(n a)^{d+1} \cdot z$ is contained in $F^{0} \mathbf{G}_{r}(X)=0$, so $(n a)^{d+1} \cdot z=0$.

Corollary 2.5. The same result holds for $K$-theory when $X$ is regular.

Corollary 2.6. The maps

$$
\begin{aligned}
B_{\mathcal{E}}^{(s / s+1)}: F^{(s / s+1)} \mathbf{G}_{r}(X) & \rightarrow F^{(s / s+1)} \mathbf{G}_{r}(X ; \mathcal{A}), \\
F: F^{(s / s+1)} \mathbf{G}_{r}(X ; \mathcal{A}) & \rightarrow F^{(s / s+1)} \mathbf{G}_{r}(X)
\end{aligned}
$$

have torsion kernels and cokernels of exponent at most na. 
Proof. This follows from the proof of the theorem.

Corollary 2.7. For any commutative ring $R$ in which na is invertible, the maps

$$
\begin{gathered}
B_{\mathcal{E}}^{z}: z_{s}(X, * ; R) \rightarrow z_{s}(X, * ; \mathcal{A} ; R), \\
B_{\mathcal{E}}: \mathbf{G}_{r}(X ; R) \rightarrow \mathbf{G}_{r}(X ; \mathcal{A} ; R), \\
F: z_{s}(X, * ; \mathcal{A} ; R) \rightarrow z_{s}(X, * ; R), \\
F: \mathbf{G}_{r}(X ; \mathcal{A} ; R) \rightarrow \mathbf{G}_{r}(X ; R)
\end{gathered}
$$

are isomorphisms.

It is interesting that this method proves the isomorphisms by means of an isomorphism of cycle complexes, not just a quasi-isomorphism.

\section{ACKNOWLEDGMENTS}

The author thanks Christian Haesemeyer, Roozbeh Hazrat, and Ray Hoobler for conversations and the referee, who made several useful suggestions for improving the exposition.

\section{REFERENCES}

1. Benjamin Antieau, Cohomological obstruction theory for Brauer classes and the period-index problem, J. K-Theory 8 (2010), no. 3, 419-435. MR2863419

2. G. Cortiñas and C. Weibel, Homology of Azumaya algebras, Proc. Amer. Math. Soc. 121 (1994), no. 1, 53-55. MR.1181159 (94g:16009)

3. William G. Dwyer and Eric M. Friedlander, Etale K-theory of Azumaya algebras, Proceedings of the Luminy conference on algebraic K-theory (Luminy, 1983), vol. 34, 1984, pp. 179-191. MR772057 (86j:18009)

4. Thomas Geisser, Motivic cohomology over Dedekind rings, Math. Z. 248 (2004), no. 4, 773794. MR 2103541 (2006c:14026)

5. S. Green, D. Handelman, and P. Roberts, K-theory of finite dimensional division algebras, J. Pure Appl. Algebra 12 (1978), no. 2, 153-158. MR0480698 (58:852)

6. Alexander Grothendieck, Le groupe de Brauer. I. Algèbres d'Azumaya et interprétations diverses, Dix Exposés sur la Cohomologie des Schémas, North-Holland, Amsterdam, 1968, pp. 46-66. MR0244269 (39:5586a)

7. R. Hazrat, Reduced K-theory of Azumaya algebras, J. Algebra 305 (2006), no. 2, 687-703. MR2266848(2007i:19002)

8. R. Hazrat and R. Hoobler, K-theory of Azumaya algebras over schemes, arXiv e-prints (2009), 0911.1406. To appear in Communications in Algebra.

9. Roozbeh Hazrat and Judith R. Millar, A note on K-theory of Azumaya algebras, Comm. Algebra 38 (2010), no. 3, 919-926. MR2650377 (2011c:19007)

10. I. N. Herstein, Noncommutative rings, The Carus Mathematical Monographs, No. 15, Mathematical Association of America, 1968. MR0227205 (37:2790)

11. Bruno Kahn and Marc Levine, Motives of Azumaya algebras, J. Inst. Math. Jussieu 9 (2010), no. 3, 481-599. MR2650808 (2011j:19005)

12. Robert W. Thomason, Algebraic K-theory and étale cohomology, Ann. Sci. École Norm. Sup. (4) 18 (1985), no. 3, 437-552. MR826102 (87k:14016)

Department of Mathematics, University of California Los Angeles, 520 Portola Plaza, Los Angeles, California 90095

E-mail address: antieau@math.ucla.edu 